\title{
X-ray Holography with a Position Sensitive Detector
}

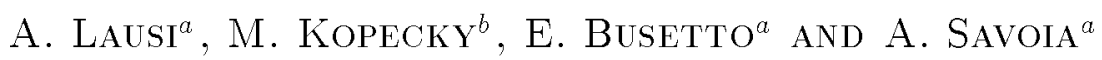 \\ ${ }^{a}$ Sincrotrone Trieste, Strada Statale 14 - km 163.5 in Area Science Park \\ 34012 Basovizza - Trieste, Italy \\ ${ }^{b}$ Institute of Physics, Czech Academy of Sciences \\ Na Slovance 2, 18040 Prague, Czech Republic
}

\begin{abstract}
Integrating photon collection on position sensitive detectors used in combination with an absorption filter offers many advantages in X-ray fluorescence holography experiments: (i) the simultaneous recording of the full hologram allows us to overcome most of the difficulties - notably the large number of experimental points and the precision requirements - that make the practical realization of the measurement of holographic patterns very difficult and time demanding also when using powerful synchrotron radiation sources; (ii) the high angular resolution and the very precise detection of intensities make the reconstruction of images of distant as well as light atoms possible; (iii) area detectors are readily available at most crystallography dedicated beamlines at synchrotron radiation sources, so there is no need of complex, dedicated instrumentation. As an example, the hologram of a $\mathrm{CoO}$ single crystal recorded on an imaging plate is reported, evidencing the images of atoms located up to more than $7 \AA$ far from the emitter and also of the light oxygen atoms.
\end{abstract}

PACS numbers: 61.10.-i, 07.85.-m, 42.40.-i

\section{Introduction}

Real-space three-dimensional imaging at atomic resolution, the original goal that led Gabor to the discovery of holography, was not achieved for a long time. Only recently, Szöke pointed out that the interference patterns of characteristic X-rays, photoelectrons or Auger electrons emitted by atoms inside a solid can be thought of as holograms and used for resolving the vicinity of the emitting atoms [1]. And indeed shortly after Szöke argument was presented, electron emission holography was developed both theoretically and experimentally into a useful 
practical tool in surface science [2]. In spite of the more ideal nature of X-ray scattering by atoms, i.e. (i) negligible multiple scattering, (ii) much more isotropic character, (iii) negligible phase shift when compared to electrons, the development of X-ray fluorescence holography (XFH) as bulk probe has been much slower, and only in 1996 the first successful internal source XFH experiment was reported by Tegze et al. [3].

The main problem of XFH consists in the practical realization of experiments. The large number of experimental points and the precision requirements make the measurements of holographic patterns very difficult and time demanding also when using powerful synchrotron radiation sources. As a consequence only a few successful experiments on well-known structures of heavy atoms have been reported so far [3-6], and only one experiment reports imaging of light atoms [7].

We reported recently a new approach to the detection of fluorescence photons [8] which make it possible to measure higher flux and to reduce drastically measuring times in XFH experiments.

\section{Theory}

The internal source scheme of XFH $[1,3]$ is schematically shown in Fig. 1: fluorescence from a particular target atom $A$ is excited by means of a suitable ionizing radiation; the electric field $\psi(\boldsymbol{k})$ in the location of a detector positioned at some large distance $R$ from the emitter consists of the unscattered portion of the emitted wave $\psi_{0}(\boldsymbol{k})$, which serves as holographic phase reference ( $R$-wave), and of the portion elastically scattered at neighboring atoms $\psi_{\mathrm{S}}\left(\boldsymbol{k}, r_{\mathrm{j}}\right)$ (object or $O$-waves). The function $\psi_{\mathrm{S}}\left(\boldsymbol{k}, r_{\mathrm{j}}\right)$ depends on the position $r_{j}$ of the $j$-th neighboring atom, with an amplitude proportional to $1 /\left|r_{j}\right|$, and phase determined by the difference in path $k \cdot r_{j}-k r_{j}$ relative to the reference wave. The angular distribution of fluorescence intensity

$$
I(\boldsymbol{k}) \propto|\psi(\boldsymbol{k})|^{2}=\left|\psi_{0}(\boldsymbol{k})\right|^{2}+\left|\psi_{\mathrm{S}}\left(\boldsymbol{k}, \boldsymbol{r}_{j}\right)\right|^{2}+2 \operatorname{Re}\left[\psi_{0}(\boldsymbol{k}) \psi_{\mathrm{S}}^{*}\left(\boldsymbol{k}, \boldsymbol{r}_{j}\right)\right]
$$

can be measured by moving the detector on the far-field sphere. The first term, the intensity of the reference wave, represents an isotropic background. The second term, the intensity of the object wave, is negligible for hard X-rays with the exception of directions fulfilling the Bragg condition (Kossel lines). The hologram, constituted by the interference between the reference and object waves, is defined as

$$
\chi(\boldsymbol{k}) \equiv \frac{\psi_{\mathrm{S}}^{*}\left(\boldsymbol{k}, \boldsymbol{r}_{j}\right)}{\psi_{0}^{*}(\boldsymbol{k})}+\frac{\psi_{\mathrm{S}}\left(\boldsymbol{k}, \boldsymbol{r}_{j}\right)}{\psi_{0}(\boldsymbol{k})} .
$$

This ratio depends on the wave vector $k$ and on the environment of the emitter, i.e. on the positions and the atomic weights of surrounding atoms. The extraction of the hologram from the experimental data is not easy because of its magnitude: $\chi(k) \approx 10^{-4}-5 \times 10^{-3}$. Comprehensive reviews on the data-treatment procedure 


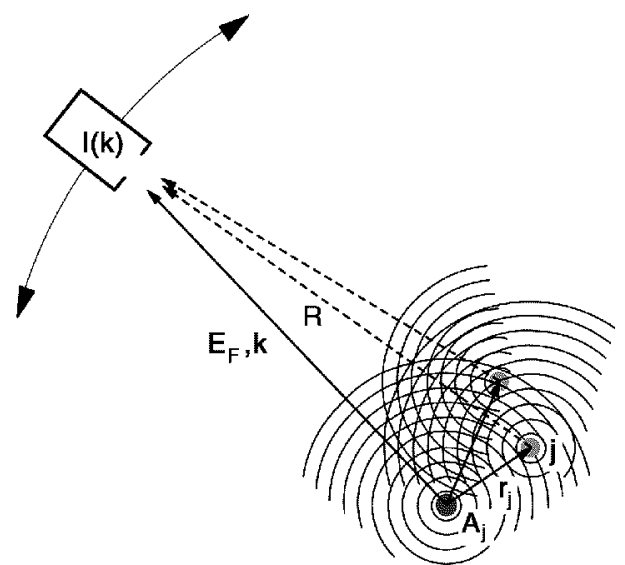

Fig. 1. The internal source X-ray fluorescence holography scheme: fluorescence from a. particular target atom $A$, approaching a detector located at some large distance $R$, constitutes the holographic reference beam, with energy $E_{\mathrm{F}}$ and wave vector $k$. In other directions the fluorescence is scattered by neighboring atoms at positions $\boldsymbol{r}_{j}$. A part of this singly scattered fluorescence, the holographic object beam, will travel directly towards the detector and interfere with the reference beam. By moving the detector on a far-field sphere the resulting holographic pattern of intensities $I(k)$ can be recorded, normalized and transformed into a real space holographic image.

required can be found in the literature [9]. The real-space reconstruction $U(\boldsymbol{r})$ can be calculated from $\chi(\boldsymbol{k})$ using the Helmholz-Kirchhoff theorem

$$
U(\boldsymbol{r})=\iint \chi(\boldsymbol{k}) \exp (-\mathrm{i} \boldsymbol{k} r) \mathrm{d} \Omega_{\boldsymbol{k}},
$$

where the integral is over the part of a sphere in reciprocal space with radius $k=|\boldsymbol{k}|$ for which intensities $I(\boldsymbol{k})$ have been measured.

In practice, always a large number of atoms is excited. Different atoms emit the fluorescence waves with random phases and do not interfere. Their intensities simply add up at the detector. As a consequence, the overall signal is simply the sum of the individual holograms and the reconstructed image represents the mean environment of all emitters.

\section{Experiment}

The angular region on which the fluorescence intensity has to be recorded - ideally, the full sphere around the sample - can be reduced by using the symmetry of the sample structure. In the case of crystals with a cubic unit cell, at least the signal in a cone with an aperture of about 56 degrees has to be measured with an angular resolution of the order of 1 degree [9]. Therefore, the number of required picture elements is in the order of a few thousand, and for each of 
them at least $10^{7}-10^{8}$ photons have to be collected in order to achieve sufficient statistics. In previously reported experiments the elastically scattered primary beam, inelastic scattering as well as characteristic $K_{\beta}$ line emitted by a reference atom were suppressed by means of a curved graphite crystal analyzer $[4,6]$ and/or by an energy dispersive detector $[3,5]$. But selecting such a narrow window around the emitter characteristic $K_{\alpha}$ line, determined either by the rocking curve of the monochromator or by the energy resolution of the photon counting detector, is accompanied with intensity loss. The photon count rate is strongly reduced because of the low reflectivity of the graphite crystal, or by the linearity limit of energy dispersive detectors, and eventually also because of the small detection solid angle.

The point is that it is not necessary to select such a narrow energy window. As reported elsewhere [10] we have already performed a successful XFH experiment using a properly chosen absorption filter in order to effectively discriminate the fluorescence signal, rejecting the scattered part of the primary beam. We extend here this concept, exploiting the energy selectivity of a foil filter in conjunction with the use of an integrating position sensitive detector.

The experiment, already reported in [8], has been carried out at the diffraction beamline of the ELETTRA synchrotron radiation source in Trieste with the experimental setup shown in Fig. 2, utilizing the same mar345 imaging-plate detector system, which is normally used for single-crystal diffraction data collection. The sample, a slab of a $\mathrm{CoO}$ single crystal with surfaces parallel to the (001)

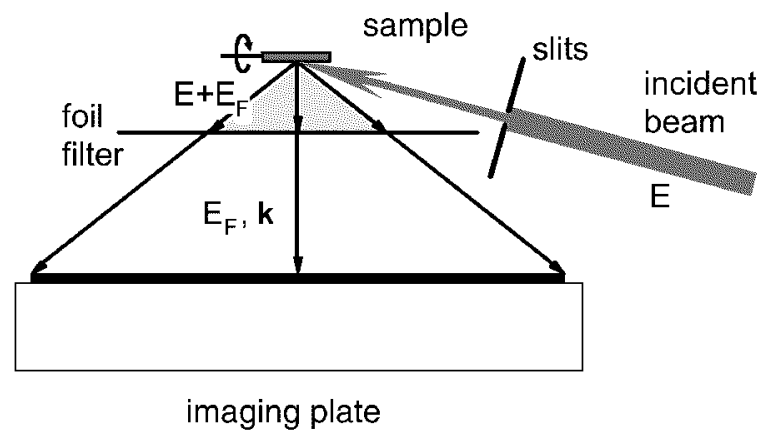

Fig. 2. Experimental setup for XFH with area detector: the elastically scattered primary beam with energy $E$ is absorbed by a foil filter and only the fluorescence photons, with energy $E_{\mathrm{F}}$, reach the detector.

crystallographic plane, was mounted on the built-in single axis goniometer. The energy of the incident beam was selected with a double-crystal Si(111) monochromator at $8.0 \mathrm{keV}$, slightly above the cobalt $K$ absorption edge $(7.709 \mathrm{keV})$. A $50 \mu \mathrm{m}$ thick iron absorber* was placed between the sample and the detector.

*Iron, whose $K$ absorption edge is at $7.112 \mathrm{keV}$, is evidently a suitable material for discriminating the cobalt $K_{\alpha}$ characteristic line. 
The elastically scattered primary beam as well as the cobalt $K_{\beta_{1}}$ characteristic line $(7.649 \mathrm{keV})$ [11] from the sample were absorbed in the iron foil. Thus only photons corresponding to the cobalt $K_{\alpha_{1,2}}$ doublet with energies $6.930 \mathrm{keV}$ and $6.915 \mathrm{keV}$, respectively, along with some inelastically scattered photons reached the detector, located at the distance of $80 \mathrm{~mm}$. The transmission of the foil filter is in fact as low as $0.00061 \%$ and $0.00015 \%$, respectively at $8 \mathrm{keV}$ and at the Co $K_{\beta_{1}}$ line, and rises to more than $10 \%$ at the energy of the cobalt $K_{\alpha_{1,2}}$ doublet. A pixel size of $150 \times 150 \mu \mathrm{m}^{2}$ was selected by the read-out software. In this configuration, the intensities of the fluorescence emitted in more than 4,000,000 directions inside the cone of $\pm 65^{\circ}$ around the normal to the sample surface were recorded at the same time. The total photon flux incident on the whole imaging plate was approximately $5 \times 10^{8}$ photons/s which, for example, represents a gain of about three orders of magnitude in comparison to the XFH experiments with a fast solid state detector. The dynamic range of the image plate is approximately 1:100,000 photons/pixel with a background of 20 photons/pixel. 64 images taken with $50 \mathrm{~s}$ exposure time were summed in order to improve statistics. The precision of experimental data of the order of $10^{-3}\left(10^{-4}\right)$ with the angular resolution better than $0.1^{\circ} \times 0.1^{\circ}\left(1.0^{\circ} \times 1.0^{\circ}\right)$ was achieved in this way. The typical image collected is shown in Fig. $3 \mathrm{a}$.
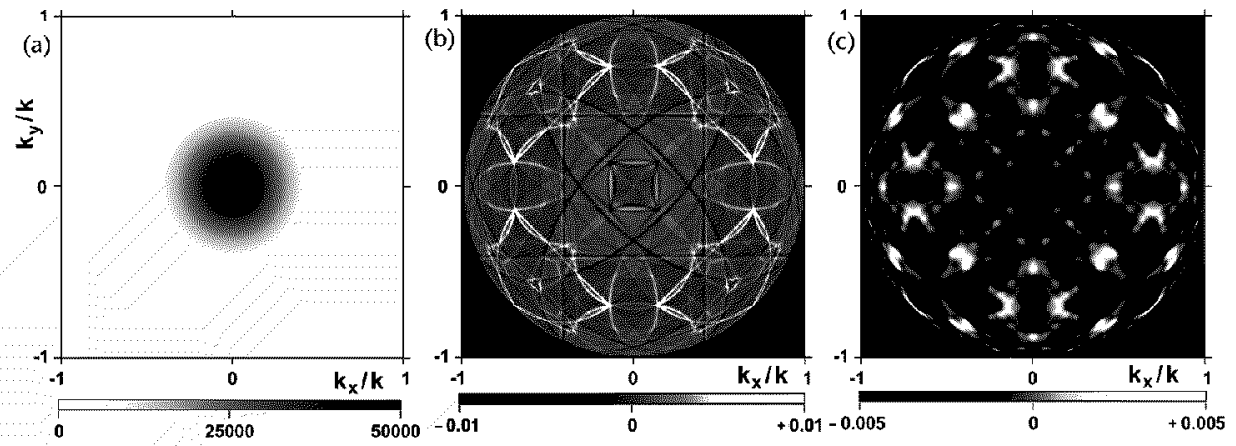

Fig. 3. (a) The typical raw data image collected at the detector; the intensity scale is reported in the bottom bar; (b) the result of the background removal, showing the Kossel lines superimposed to the hologram; (c) the hologram, obtained from (b) by canceling the sharp features applying a low-pass filter to the image. The $x$ and $y$ axes are along the $[100]$ and $[010]$ directions, respectively.

Recording the whole image on the position sensitive detector eliminates the need to normalize the data to the flux of the incident beam, a prominent source of statistical errors inherent to all experiments collecting the hologram point by point. The experimental data were corrected for the variations in the thickness of the absorber and in the efficiency of individual imaging plate pixels by dividing each image by a second image recorded while rotating the crystal around an 
axis laying on the sample surface, randomly oriented with respect to the crystallographic axes. The fluorescence background, which is modulated by the angular dependence of the absorption, was removed by means of a $25^{\circ}$ wide high-pass filter. The resulting image, containing only the symmetry-related sharp features (Kossel lines), deriving from the crystalline nature of the sample, and the hologram, is shown in Fig. 3b, in which the Kossel lines were used to extend the data to the full sphere [6]. These are then filtered out numerically using the combination of a median and a Savitzky-Golay low pass filter. The width of both filters was chosen to be approximately $2^{\circ}$ in order to image the real-space region around the emitter with the diameter of about $8 \AA$. The resulting hologram is shown in Fig. 3c. As an example, the reconstruction of the (001) crystallographic plane at $z=a$ is illustrated in Fig. 4a. In contrast to a previous experiment carried out by using
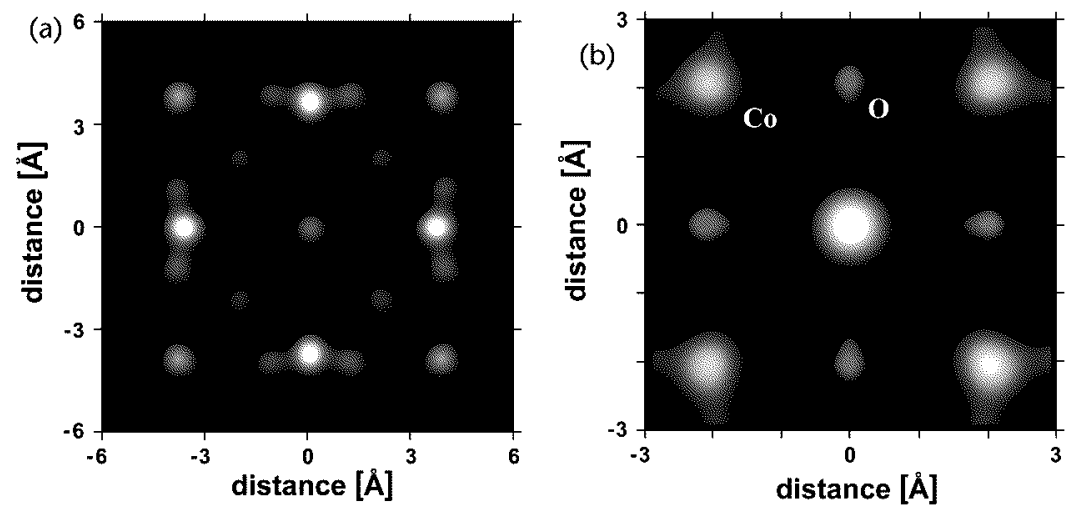

Fig. 4. (a) Reconstructed image of the (001) plane of cobalt atoms at $z=a$ (CoO has a face-centered cubic structure with a lattice constant $a=4.26 \AA$ ); (b) a detail of the same plane, shown with a different intensity scale in order to evidence the position of the oxygen atoms. The $x$ and $y$ axes are along the [100] and [010] directions, respectively.

the same sample [6], the images of the third, fourth, and fifth Co neighbors, up to a distance of more than $7 \AA$ from the emitter are clearly visible in expected positions because of the high precision and angular resolution of the data. It is possible to locate the positions of some of the light oxygen atoms, as evidenced in the enlarged view of Fig. 4b. But it has to be mentioned that the real-space image suffers from two disturbing effects: (i) the interference between the real and twin images of atoms inherent to any single-energy reconstruction [12] and (ii) the destructive effect of "tails" of images of neighboring atoms. Consequently, the intensities of images of cobalt atoms do not decrease linearly with increasing distance from the emitter in Fig. $4 \mathrm{~b}$ and even images of some atoms are missing in reconstructions of other crystallographic planes. Nevertheless, the real-space image obtained from the experimental hologram is in very good agreement with the simulation, including relative intensities of the reconstructed atoms. 


\section{Conclusion}

It is obvious that experimental configurations with detection of photons within a narrow energy window cannot always be replaced with the method of absorption filters. Suitable thin foil filters are however available in many cases, and then the technique of absorption filters coupled to an area detector reduces drastically the duration of experiments and makes it possible to measure data with better statistics. The improvement of data precision allows the recording of holographic signals from weaker scatterers and to get images of lighter atoms. For example, the method of absorption filters could be used for most of the samples used in XFH experiments performed up to now.

Moreover, internal source XFH is the only strategy allowing to collect data in a non-sequential way and, as demonstrated, can lead to comparable results in much shorter times and by using a simple non-dedicated experimental setup. The combination of this strategy with fast area detectors and ultra-bright sources of X-rays (as, for example, free-electron lasers) would make X-ray fluorescence holography a promising tool for imaging of structures of small clusters or molecules and/or time-resolved studies.

The technique described in the paper uses an internal source concept of holography. It means, in principle, that holograms can be obtained only for a few characteristic energies of atoms present in the sample and it can be insufficient for removing twins and other artefacts related to the Helmholtz-Kirchhoff reconstruction algorithm. However, the method of fast X-ray holography can provide useful information on a sample structure in spite of the fact that a very limited number of a vailable energies will not always allow to determine unambigously positions of all atoms (especially in the case of non-centrosymetric crystals).

\section{References}

[1] A. Szöke, in: Short Wavelength Coherent Radiation: Generation and Applications, Eds. T. Attwood, J. Boker, AIP Conf. Proc., Vol. 147, AIP, New York 1986, p. 361.

[2] For a review on the argument, see C.S. Fadley, Surf. Sci. Rep. 19, 231 (1993).

[3] M. Tegze, G. Faigel, Nature 380, 49 (1996).

[4] T. Gog, P.M. Len, G. Materlik, D. Bahr, C.S. Fadley, C. Sanchez-Hanke, Phys. Rev. Lett. 76, 3132 (1996).

[5] B. Adams, D.V. Novikov, T. Hiort, G. Materlik, Phys. Rev. B 57, 7526 (1998).

[6] M. Tegze, G. Faigel, S. Marchesini, M. Belakhovsky, A.I. Chumakov, Phys. Rev. Lett. 82, 4847 (1999).

[7] M. Tegze, G. Faigel, S. Marchesini, M. Belakhovsky, O. Ulrich, Nature 407, 38 (2000).

[8] M. Kopecky, E. Busetto, A. Lausi, M. Miculin, A. Savoia, Appl. Phys. Lett. 78, 2985 (2001).

[9] See, for example, G. Faigel, M. Tegze, Rep. Prog. Phys. 62, 355 (1999). 
[10] E. Busetto, M. Kopecky, A. Lausi, R.H. Menk, M. Miculin, A. Savoia; Phys. Rev. $B$ 62, 5273 (2000).

[11] B.L. Henke, E.M. Gullikson, J.C. Davis, Atomic Data and Nuclear Data Tables 54, 181 (1993).

[12] P.M. Len, S. Thevuthasan, C.S. Fadley, A.P. Kaduwela, M.A. Van Hove, Phys. Rev. B 50, 11275 (1994). 